\title{
Evaluation of Index of Cardio-Electrophysiological Balance in Patients With Atrial Fibrillation on Antiarrhythmic-Drug Therapy
}

\author{
Abdulmecit Afsin ${ }^{\mathrm{a}, \mathrm{e}}$, Ramazan Asoglu ${ }^{\mathrm{a}}$, Mehmet Ali Kobat ${ }^{\mathrm{b}}$, \\ Emin Asoglu ${ }^{\mathrm{c}}$, Arif Suner ${ }^{\mathrm{d}}$
}

\begin{abstract}
Background: Index of cardio-electrophysiological balance (iCEB) has been described as a novel risk marker for predicting malignant ventricular arrhythmia. There remains limited evidence on the effects of amiodarone and propafenone used for sinus rhythm maintenance on iCEB in patients with atrial fibrillation (AF). The aim of this study was to evaluate $\mathrm{iCEB}$ in patients with $\mathrm{AF}$ on antiarrhythmic-drug therapy.

Methods: A total of 108 patients with AF (68 patients using amiodarone and 40 patients using propafenone) and 50 healthy subjects were included in the study. All groups underwent a standard 12-lead surface electrocardiogram. QRS duration, QT, T wave peak-to-end (Tp-e) intervals, iCEB (QT/QRS) and iCEBc (heart rate-corrected $\mathrm{QT}(\mathrm{QTc}) / \mathrm{QRS})$ rates were calculated from the electrocardiogram and compared between groups.

Results: QT, Tp-e intervals and Tp-e/QT ratio were significantly longer in the amiodarone group than the propafenone and control groups $(\mathrm{P}<0.001$, for all). iCEB was similar in the amiodarone and control groups $(4.4 \pm 0.6$ and $4.2 \pm 0.4 ; \mathrm{P}>0.05)$, while $\mathrm{iCEB}$ values in the propafenone group were significantly lower than the amiodarone group and control groups $(3.9 \pm 0.5 ; \mathrm{P}<0.001)$. There was a significantly difference in $\mathrm{iCEBc}$ values among the amiodarone, control and propafenone groups $(4.8 \pm 0.6,4.6 \pm 0.4$ and $4.3 \pm 0.6 ; \mathrm{P}<0.001$, respectively).
\end{abstract}

Conclusions: In this study, higher iCEBc parameters were observed

Manuscript submitted October 22, 2020, accepted November 9, 2020

Published online December 11, 2020

aDepartment of Cardiology, Adiyaman Training and Research Hospital, Adiyaman, Turkey

${ }^{b}$ Department of Cardiology, Firat University Faculty of Medicine, Elazig, Turkey

'Department of Cardiology, Mardin State Hospital, Mardin, Turkey

dDepartment of Cardiology, Adiyaman University Faculty of Medicine, Adiyaman, Turkey

${ }^{e}$ Corresponding Author: Abdulmecit Afsin, Department of Cardiology, Adiyaman University Training and Research Hospital, Adiyaman 02000, Turkey. Email: abdulmecitafsin@gmail.com

doi: https://doi.org/10.14740/cr1185 in patients using amiodarone, while iCEBc values were lowest among patients with AF using propafenone. Further studies are needed to determine whether these electrophysiological changes are associated with ventricular arrhythmias for patients with AF on antiarrhythmicdrug therapy.

Keywords: Atrial fibrillation; Antiarrhythmic-drug; Index of cardioelectrophysiological balance; Ventricular arrhythmia

\section{Introduction}

Atrial fibrillation (AF) is the most common heart rhythm disorder in clinical practice and is a significant risk factor for stroke and heart failure [1]. The prevalence of $\mathrm{AF}$ in the general population is $1-2 \%$, and its incidence and prevalence increase with age. In older adults aged 65 years and older, the prevalence of $\mathrm{AF}$ increases to over $10 \%$ [2]. In AF treatment, the goal is to reduce cardiovascular mortality and morbidity. The two basic principles of AF treatment are preventing thromboembolic events and ensuring rhythm control or controlling the ventricular rate [3]. The use of antiarrhythmic drugs (AADs) or catheter ablation is often required to maintain sinus rhythm in patients with recurrent paroxysmal $\mathrm{AF}$ and in patients with persistent AF to maintain sinus rhythm following cardioversion. The primary concerns associated with the use of AADs are proarrhythmia and consequent ventricular tachyarrhythmia as well as bradycardia [3]. Regular 12-lead electrocardiogram (ECG) monitoring is required to assess the risk for proarrhythmia in patients taking AADs. Current guidelines recommend careful evaluation of the heart rhythm, heart rate, QRS duration, QT interval (i.e., the period of ventricular depolarization and repolarization) and QTc interval (i.e., QT interval corrected for heart rate) in ECG [4]. Despite their poor predictive value, QT interval and QRS duration are widely used in the clinical practice to assess the risk for drug-induced ventricular arrhythmia and sudden cardiac death (SCD).

Many ECG parameters have been developed in an effort to predict ventricular arrhythmias. One example is transmural dispersion of repolarization (TDR), which is measured using the $\mathrm{T}$ wave peak-to-end ( $\mathrm{Tp}-\mathrm{e})$ interval and $\mathrm{Tp}$-e/QT ratio. TDR is a potential marker for ventricular arrhythmias and 
SCD in patients with acquired long QT syndrome, congenital long QT syndrome, Brugada syndrome, acute myocardial infarction and heart failure [5-7]. Recently, the index of the cardio-electrophysiological balance (iCEB), which is calculated as QT interval divided by QRS duration (QT/QRS), was identified as a potential marker for predicting drug-induced ventricular arrhythmias in an animal model [8]. iCEB is equivalent to the cardiac wavelength $\lambda$, which plays an important role in ventricular arrhythmias and is measured via invasive electrophysiology (EP). Previous studies have suggested that iCEB may offer a non-invasive and readily measurable marker to detect increased arrhythmic risk in patients [9]. To date, there remains limited evidence on the effects of amiodarone and propafenone used for sinus rhythm maintenance on iCEB in patients with AF. This study assessed the iCEB and TDR values in AF patients on amiodarone and propafenone therapy.

\section{Materials and Methods}

\section{Study population}

The study included patients who attended the cardiology outpatient clinic of two different centers between January 2019 and December 2019. Eligible patients included those who were using propafenone (150 $\mathrm{mg}$ three times daily) or amiodarone (200 mg once a day) for paroxysmal or persistent AF with sinus rhythm detected in ECG. The control group included healthy individuals with sinus rhythm assessed using ECGs. Volunteers were recruited from hospital staffs. The healthy controls who had diagnosed cardiac or other organic disease, or who were using medications, were excluded.

Paroxysmal AF was defined as AF that developed suddenly and ended spontaneously within 7 days, whereas persistent AF was defined as AF with medicated or electrical cardioversion lasting more than 7 days [10]. Hypertension (HT) was defined as blood pressure $\geq 140 / 90 \mathrm{~mm} \mathrm{Hg}$ or receiving antihypertensive treatment. Diabetes mellitus (DM) was defined as fasting blood glucose level $\geq 126 \mathrm{mg} / \mathrm{dL}$ or known DM diagnosis. Coronary artery disease (CAD) was defined as the presence of an angiographic lesion occupying $\geq 50 \%$ of the coronary artery, a history of coronary bypass surgery, or percutaneous coronary intervention. Stroke was diagnosed on the identification of ischemia or bleeding in the brain through clinical assessments and imaging methods in patients presenting with neurological dysfunction whose symptoms lasted more than $24 \mathrm{~h}$. Transient ischemic attack was defined as temporary neurological dysfunction that lasted less than $24 \mathrm{~h}$, which caused symptoms but did not result in death or disability [11]. Vascular disease diagnoses included $\mathrm{CAD}$, peripheral artery disease, or aortic plaque. The $\mathrm{CHA}_{2} \mathrm{DS}_{2}$-VASc (congestive heart failure/left ventricular dysfunction, HT, aged $\geq 75$ years, $\mathrm{DM}$, stroke/transient ischemic attack/systemic embolism, vascular disease, aged 65 - 74 years, sex category) scores of patients were recorded [4].

We excluded patients with the following cardiac conditions: permanent AF, long-standing persistent $\mathrm{AF}$, atrial flutter, previous AF ablation, acute decompensated heart failure and hereditary long QT syndrome. Also excluded were patients who were using medications that could affect the QRS, QT and Tp-e interval, including antibiotics, tricyclic antidepressants, antihistamines, or antipsychotics; patients with implantable cardioverter-defibrillators; those with previously known branch block and atrioventricular nodal block; and those with negative and/or biphasic T wave on their ECGs. Non-cardiac exclusion criteria were the presence of any severe non-cardiac illness limiting life expectancy, pregnancy, breast-feeding, a calculated glomerular filtration rate of $<60 \mathrm{~mL} / \mathrm{min}$ at baseline, patients with liver failure and patients with thyroid gland disease. Patients who developed liver and/or thyroid disease due to amiodarone use were included in the study. The study methodology complied with the Declaration of Helsinki and the study protocol was approved by the Institutional Ethics Committee of Adiyaman University (26/06/2019, 2019/5-12).

\section{ECG and echocardiographic examination}

All participants underwent a 12-lead ECG (CardioFax S; Nihon Kohden, Tokyo, Japan) while at rest in the supine position. The ECG was set to the paper speed of $50 \mathrm{~mm} / \mathrm{s}$ and calibrated such that $10 \mathrm{~mm}$ equals $1 \mathrm{mV}$. During the ECG recordings, all of the participants were in sinus rhythm. Resting heart rate was measured using the ECG data. ECG measurements of QRS duration, QT intervals and Tp-e intervals were manually calculated by two cardiologists who were blinded to patient data using calipers and a magnifying glass to decrease measurement errors. The measurements were performed on lead II and lead V5, and the longest QT interval and QRS complex duration were used for the analyses. The QT interval was measured from the beginning of the QRS complex to the end of the T wave, and the QT interval was corrected for heart rate using the Bazett formula: $\mathrm{QTc}=\mathrm{QT} \sqrt{ }(\mathrm{R}-\mathrm{R}$ interval). Using these measurements, Tp-e/ QT, Tp-e/QTc, QT/QRS (iCEB) and QTc/QRS (iCEBc) ratios were calculated. The interobserver and intraobserver variation coefficients were $2.3 \%$ and $2.4 \%$, respectively.

All echocardiographic examinations were performed using a Vivid 5 Pro device (General Electric, Horten, Norway) with a $2.5 \mathrm{MHz}$ transducer. The measurements were performed in the left lateral decubitus position as recommended by the current American Society of Echocardiography guidelines [12], and three consecutive cycles were averaged for each parameter. Ejection fraction (EF) was calculated using the modified Simpson method. Left ventricular systolic dysfunction was defined as a left ventricular EF of $<50 \%$. Left atrium (LA) anteroposterior diameter, diastolic interventricular septum (IVS) thickness and diastolic posterior wall thickness (PWT) were measured from parasternal long-axis views using M-mode.

Laboratory findings were collected from the hospital database. Following a $12 \mathrm{~h}$ fasting period, blood samples were collected for complete blood count analyses. Aspartate aminotransferase (AST), alanine aminotransferase (ALT), creatinine, potassium and calcium levels were analyzed using the Architect c8000 Chemistry System (Abbott Diagnostics, USA) commercial kits. Thyroid-stimulating hormone (TSH) was measured using the UniCel DxI 800 Access Immunoassay System (Beckman Coulter, USA). 


\section{Statistical analyses}

Continuous variables are reported as means \pm standard deviations or medians. Categorical variables are expressed as counts and percentages. Kolmogorov-Smirnov tests and histograms were used to test for normality and to assess the distribution of the numerical variables, respectively. Demographic and clinical features, including ECG and echocardiography parameters, comorbidities, medications, risk scores and laboratory parameters, were compared between groups. One-way analysis of variance (ANOVA) and Kruskal-Wallis tests were used for intergroup comparisons when variables were normally and nonnormally distributed, respectively. Intergroup differences were evaluated using parametric and non-parametric tests including the Tukey test and the Dwass-Steel-Critchlow-Fligner test, respectively. Differences in categorical variables were assessed using the Pearson Chi-square, Fisher exact, or Fisher-FreemanHalton tests, depending on the sample size. The analyses compared patients with and without AF. For variables that showed a normal distribution, independent samples $t$-tests were used, whereas Mann-Whitney U tests were used to compare variables with non-normally distributed data. For categorical variables, the Pearson Chi-square and Fisher exact tests were used. All statistical analyses were performed using the Jamovi (Version 1.0.7) and JASP (Version 0.11.1) software programs. P values $\leq 0.05$ were considered statistically significant.

\section{Results}

A total of 173 patients were eligible for study inclusion. Patients with unanalyzable ECG $(n=6)$ were excluded. In addition, patients who were taking sotalol $(n=9)$ were excluded due to their small sample size. After exclusions, 158 participants were included in the study. Demographic characteristics, comorbid conditions and laboratory parameters are shown in Table 1. The study groups included patients with persistent AF $(\mathrm{n}=17)$, patients with paroxysmal AF $(\mathrm{n}=91)$ and healthy individuals without AF $(\mathrm{n}=50)$. Among the patients with AF, $68(63 \%)$ were using amiodarone treatment and 40 (37\%) were using propafenone treatment at baseline. There were no differences between the groups in terms of age and sex $(\mathrm{P}>0.05)$. The amiodarone treatment group included $57(83.8 \%)$ patients with paroxysmal AF and $11(16.2 \%)$ with persistent AF, whereas the propafenone treatment group included $34(85.0 \%)$ with paroxysmal AF and six (15.0\%) with persistent AF. More than half $(51.5 \%)$ of the patients in the amiodarone group had heart failure compared to only $2.5 \%$ in the propafenone group. The prevalence of CAD and stroke were significantly higher in patients using amiodarone compared to those using propafenone (both $\mathrm{P}<0.05$ ).

\section{Echocardiographic measurements, $\mathrm{CHA}_{2} \mathrm{DS}_{2}-\mathrm{VASc}$ scores and ECG parameters}

Echocardiographic measurements, $\mathrm{CHA}_{2} \mathrm{DS}_{2}$-VASc scores and ECG parameters are shown in Table 2. The amiodarone group had a significantly lower median left ventricular EF (LVEF) and higher LA diameter compared to the propafenone group. The median $\mathrm{CHA}_{2} \mathrm{DS}_{2}$-VASc score of the amiodarone group was significantly higher than that of the propafenone group $(\mathrm{P}<0.001)$. There were no significant differences in IVS thickness and PWT between the amiodarone and propafenone groups; however, the IVS thickness and PWT values were significantly higher in both treatment groups than in the control group $(\mathrm{P}<0.001)$.

QT, QTc, Tp-e and cTp-e intervals were significantly increased in the amiodarone group compared to the propafenone and control groups (all $\mathrm{P}<0.001$ ) (Fig. 1). The intervals were similar among the propafenone and control groups (Table 2). Moreover, QRS duration was higher in the propafenone group compared to the amiodarone and control groups $(\mathrm{P}<0.001)$.

The Tp-e/QT ratio was increased in patients with AF using amiodarone compared to the propafenone and control groups. It was similar between the propafenone and control groups. The findings were similar when Tp-e/QT ratio was corrected for heart rate (Fig. 2). One (1.5\%) patient using amiodarone presented with torsades de pointes (TdP), while one $(2.5 \%)$ patient using propafenone presented with ventricular tachycardia (VT) (Table 2).

iCEB was similar among the amiodarone and control groups (4.4 \pm 0.6 and $4.2 \pm 0.4$, respectively; $\mathrm{P}>0.05)$ but was significantly lower in the propafenone group than in the amiodarone and control groups $(4.4 \pm 0.6,4.2 \pm 0.4$ and 3.9 $\pm 0.5 ; \mathrm{P}<0.001)$. There was a significant difference in $\mathrm{iCEBc}$ values between amiodarone, control and propafenone groups ( $4.8 \pm 0.6,4.6 \pm 0.4$ and $4.3 \pm 0.6$, respectively; $\mathrm{P}<0.001)$, with the amiodarone group having the highest value and the propafenone group having the lowest (Fig. 3).

The median Tp-e interval and Tp-e/QT ratio were higher in patients with AF $(n=108)$ than in controls $(\mathrm{P}<0.001$ and $\mathrm{P}=0.001$, respectively). However, there were no differences in $\mathrm{iCEB}$ and $\mathrm{iCEBc}$ values between patients with $\mathrm{AF}$ and the healthy controls ( $\mathrm{P}=0.74$ and $\mathrm{P}=0.60$, respectively) (Table 3 ).

\section{Discussion}

The study included patients with persistent and paroxysmal AF and who were taking amiodarone or propafenone therapy. To assess the effects of amiodarone and propafenone treatment on ECG parameters, resting ECGs were obtained and compared inter se and with healthy controls. Significant prolongation of TRD markers such as Tp-e interval, cTp-e interval, Tp-e/QT and cTp-e/QT ratios was observed in patients using amiodarone, compared to those in the propafenone and control groups. Among patients taking amiodarone, the $\mathrm{iCEBc}$ values were significantly higher than those in the propafenone and control groups. iCEB and $\mathrm{iCEBc}$ values were significantly lower in the propafenone group than in the amiodarone and control groups. To our knowledge, the present study is the first in the literature that evaluates the $\mathrm{iCEB}$ and $\mathrm{iCEBc}$ in patients with AF using amiodarone or propafenone for the maintenance of sinus rhythm.

In several large-scale and randomized studies, rhythm con- 
Table 1. Demographic Features, Comorbidities, Drug Use and Some Laboratory Parameters of the Amiodarone, Propafenone and Control Groups

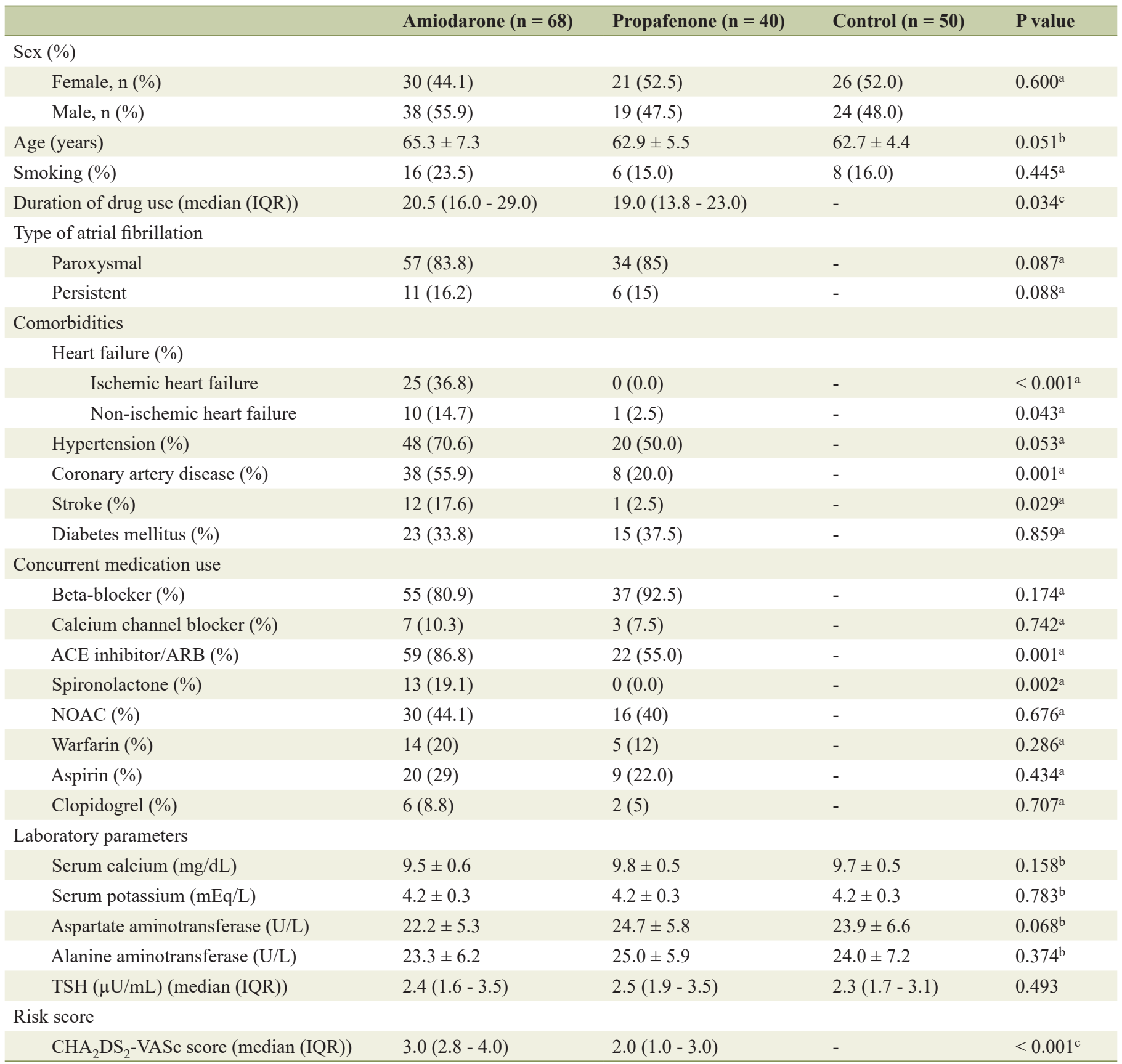

aDepending on the expected count, Pearson Chi-square, Fisher exact or Fisher-Freeman-Halton test was used. Descriptive statistics were presented as number (\%). ${ }^{b}$ One-way ANOVA was used. Descriptive statistics were presented as mean \pm standard deviation. ${ }^{\mathrm{C}}$ Mann-Whitney $\mathrm{U}$ test was used. Descriptive statistics were presented as median (IQR). ACE: angiotensin-converting enzyme; ARB: angiotensin receptor blocker; NOAC: new oral anticoagulants; TSH: thyroid-stimulating hormone; IQR: interquartile range.

trol and rate control strategies have been evaluated in patients with AF [13-15]. These studies have found no differences in the stroke or mortality outcomes between the two strategies. Moreover, the two strategies have shown similar results in terms of quality of life and heart failure-related hospitalizations. Although sinus rhythm is the natural heart rhythm, in these studies, no significant benefit was observed among patients who underwent a rhythm control strategy. This finding may be attributed to failing to maintain the sinus rhythm in patients after cardioversion. In addition, AADs can cause adverse cardiac and extracardiac effects, thus close clinical follow-up is recommended to monitor the risk for proarrhythmia. 
Table 2. Echocardiographic and Electrocardiographic Parameters of Amiodarone, Propafenone and Control Groups

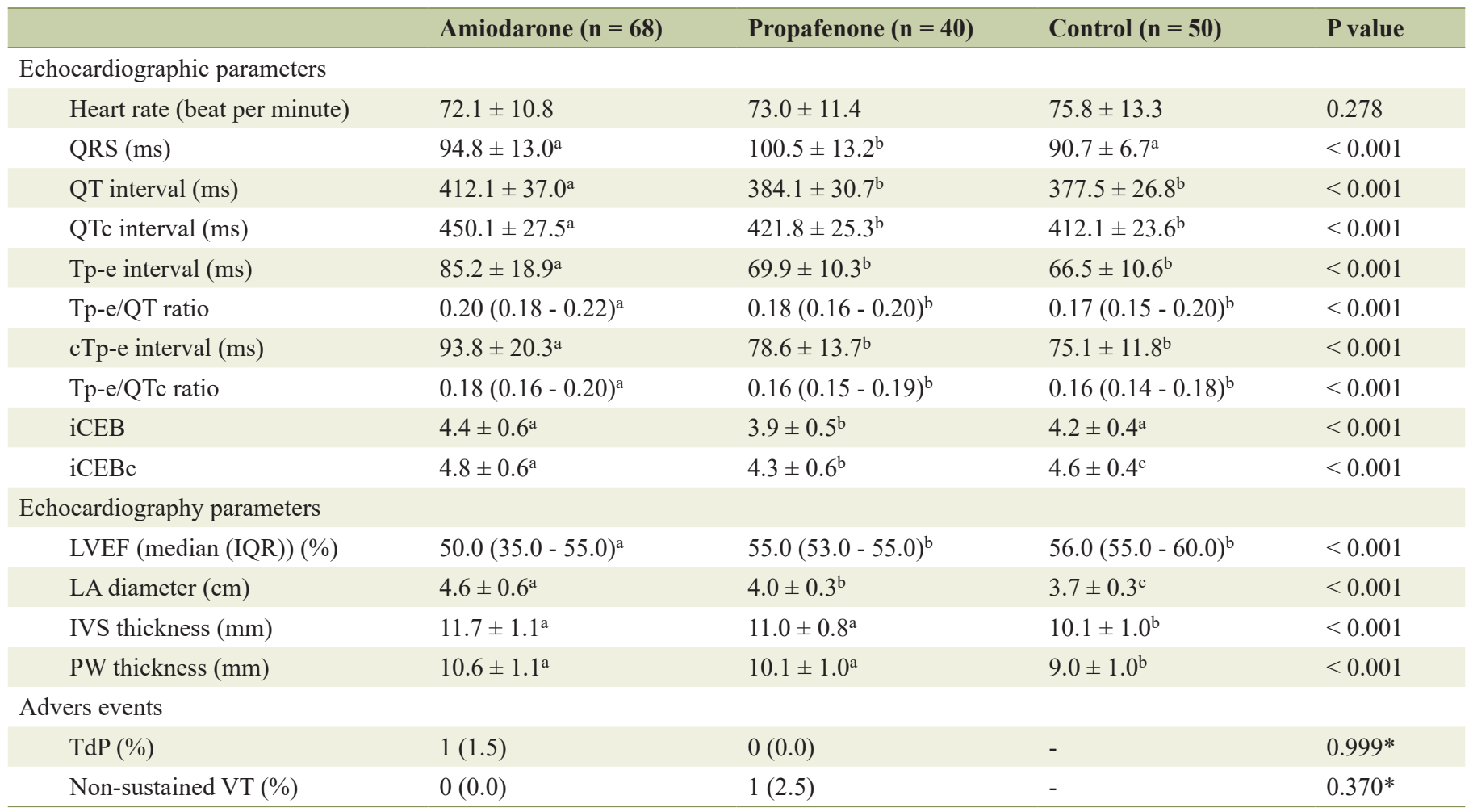

${ }^{*}$ Depending on the expected count, Pearson Chi-square, Fisher exact or Fisher-Freeman-Halton test was used. Descriptive statistics were presented as number (\%). Multiple comparisons were marked with superscript letters in the table. As same letters notify no significant difference between the groups, different letters mean a significant difference between the groups. Tp-e: T wave peak-to-end; iCEB: index of cardio-electrophysiological balance; iCEBc: corrected iCEB; LVEF: left ventricular ejection fraction; LA: left atrial; IVS: interventricular septum; PW: posterior wall; VT: ventricular tachycardia; TdP: torsades de pointes; Tp-e: transmural dispersion of repolarisation; cTp-e: corrected Tp-e.

In clinical practice, $\mathrm{Na}^{+}$channel blockers (class IC) such as flecainide and propafenone, and $\mathrm{K}^{+}$channel blockers (class III) such as amiodarone, dronedarone, dofetilide and sotalol are often used as part of a rhythm control strategy in patients with AF. A recent European survey found that beta-blockers, propafenone, flecainide and amiodarone were most often used as first-line AADs for the prevention of AF in several patient groups [16]. Amiodarone is a broad-spectrum antiarrhythmic medication that acts on $\mathrm{K}^{+}$ion channels throughout the heart. The medication acts on the rapidly delayed rectifier potassium

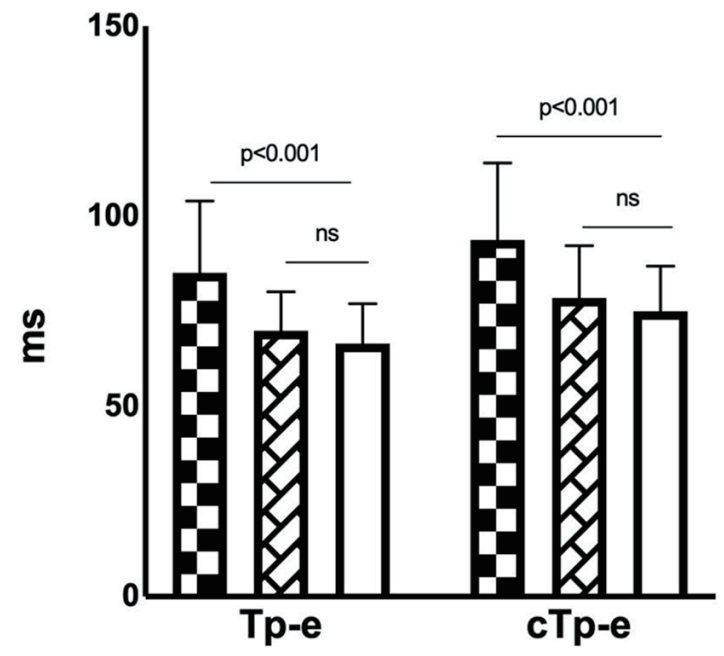

-7 Amiodarone

Z Propafenone

$\square$ Control

Figure 1. Tp-e and cTp-e intervals in drug and control groups. Tp-e: T wave peak-to-end; cTp-e: corrected Tp-e. 


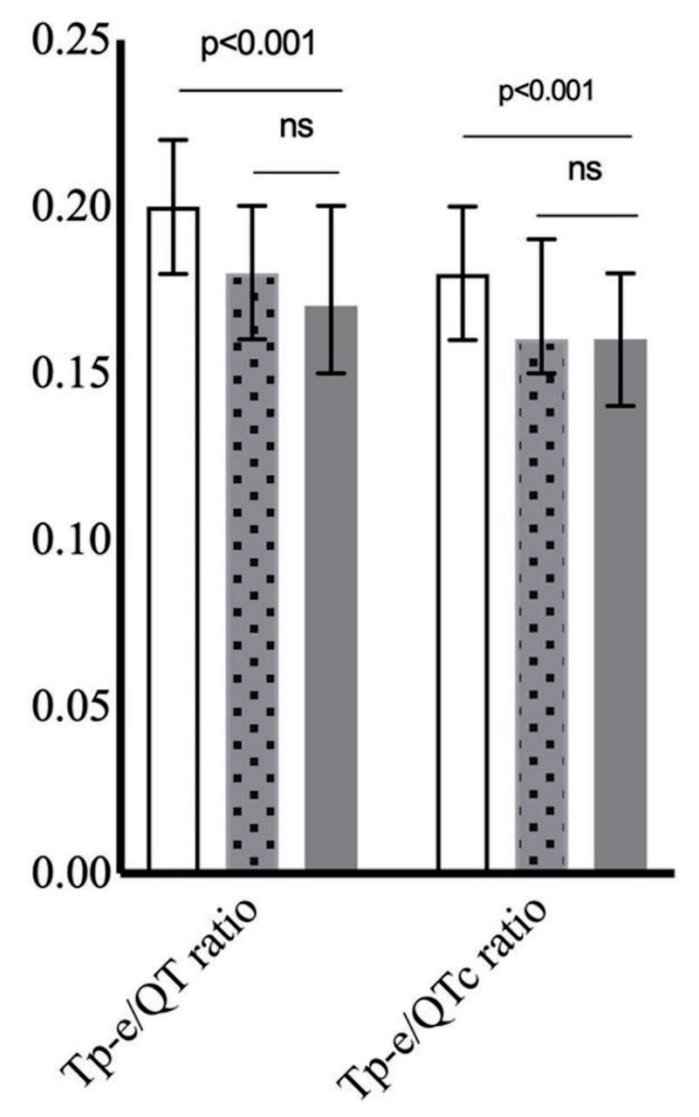

Amiodarone

Propafenone

Control

Figure 2. Tp-e/QT and Tp-e/QTc rates in drug and control groups. Tp-e: T wave peak-to-end; Tp-e/QTc: corrected Tp-e/QT.

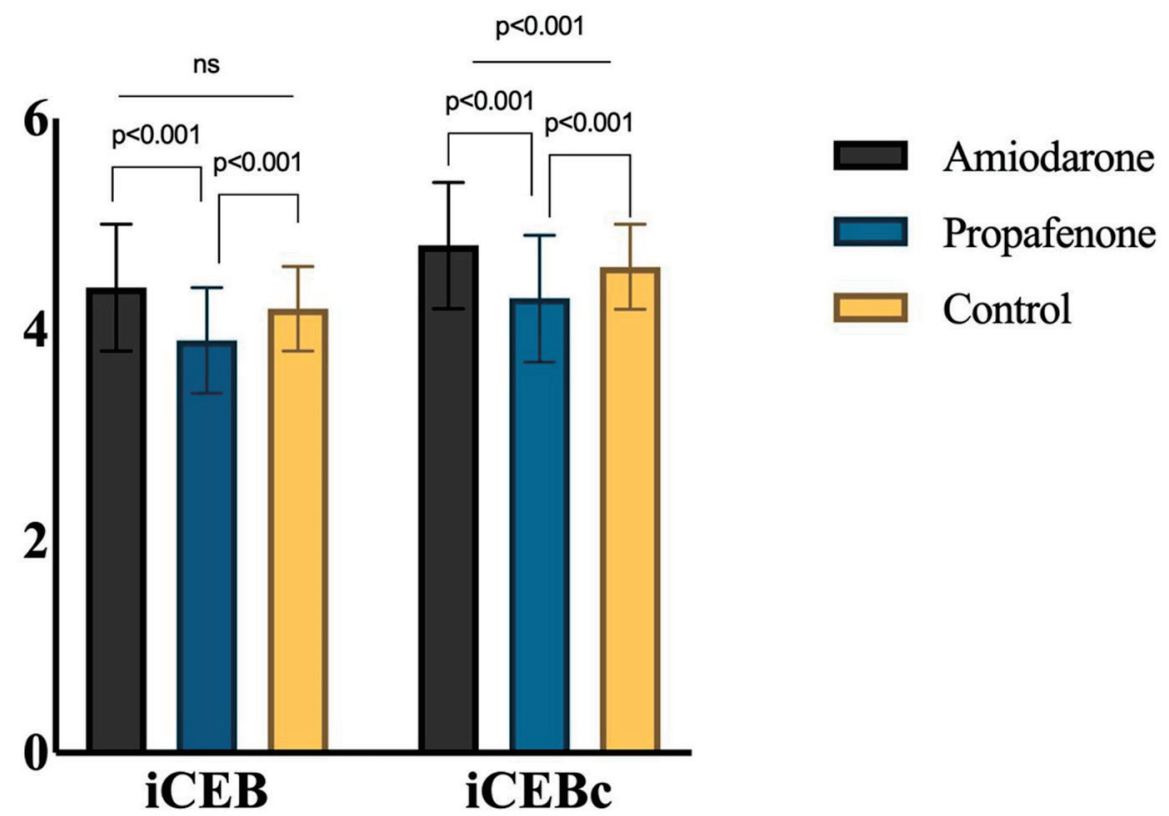

Figure 3. iCEB and iCEBc values in drug and control groups. iCEB: index of cardio-electrophysiological balance; iCEBc: corrected $\mathrm{iCEB}$. 
Table 3. Demographic Features, Electrocardiographic and Echocardiographic Parameters of the Whole Atrial Fibrillation and Control Subjects

\begin{tabular}{|c|c|c|c|}
\hline & Atrial fibrillation $(n=108)$ & Control $(n=50)$ & P value \\
\hline $\operatorname{Sex}(\%)$ & & & 0.698 \\
\hline Female, n (\%) & $51(47.2)$ & $26(52.0)$ & \\
\hline Age (years) & $64.4 \pm 6.7$ & $62.7 \pm 4.4$ & 0.112 \\
\hline Smoking (\%) & $22(20.4)$ & $8(16.0)$ & 0.515 \\
\hline Heart rate (beats per minute) & $72.4 \pm 10.9$ & $75.8 \pm 13.3$ & 0.121 \\
\hline QRS (ms) & $96.9 \pm 13.3$ & $90.7 \pm 6.7$ & $<0.001$ \\
\hline QT interval (ms) & $401.7 \pm 37.2$ & $377.5 \pm 26.8$ & $<0.001$ \\
\hline QTc interval (ms) & $439.6 \pm 29.9$ & $412.1 \pm 23.6$ & $<0.001$ \\
\hline Tp-e interval (ms) & $79.5 \pm 17.8$ & $66.5 \pm 10.6$ & $<0.001$ \\
\hline iCEB & $4.2 \pm 0.6$ & $4.2 \pm 0.4$ & 0.740 \\
\hline $\mathrm{iCEBc}$ & $4.6 \pm 0.7$ & $4.6 \pm 0.4$ & 0.602 \\
\hline \multicolumn{4}{|l|}{ Echocardiography parameters } \\
\hline LVEF (median (IQR)) (\%) & $54.0(44.5-55.0)$ & $56.0(55.0-60.0)$ & $<0.001$ \\
\hline LA diameter $(\mathrm{cm})$ & $4.4 \pm 0.6$ & $3.7 \pm 0.3$ & $<0.001$ \\
\hline
\end{tabular}

iCEB: index of cardio-electrophysiological balance; iCEBc: corrected iCEB; LVEF: left ventricular ejection fraction; LA: left atrial; Tp-e: T wave peakto-end; cTp-e: corrected Tp-e.

current $\left(\mathrm{I}_{\mathrm{Kr}}\right)$, thereby delaying repolarization. Accordingly, action potential and refractory period are elongated, which results in an increased QT interval and slightly increased QRS duration in ECGs. As a class IC antiarrhythmic agent, propafenone blocks the fast $\mathrm{Na}^{+}$channels in Purkinje fibers and ventricular myocardium. Moreover, it has been shown to slightly block the $\mathrm{I}_{\mathrm{Kr}}$ and beta-adrenergic receptors. As a result, propafenone increases the QRS duration without affecting the QT interval in ECGs [17].

All AADs used to maintain sinus rhythm have the potential to induce proarrhythmia, including monomorphic VT, TdP, or ventricular fibrillation (VF). Several strategies are used to identify the risk for proarrhythmia [18]. For class III agents, if the QTc interval is $>500 \mathrm{~ms}$ or if the patient experiences an increase in the QTc interval of $>60 \mathrm{~ms}$ after medication administration, discontinuation of the drug is recommended. For class IC drugs, when patients experience an increase of $25 \%$ in QRS interval from baseline, there is an increased risk for malignant ventricular arrhythmia, and medication discontinuation is recommended $[19,20]$. Nevertheless, risk stratification for SCD from drug-induced proarrhythmia remains challenging. In the majority of drug-induced TdP cases, the QT interval was observed to be $>500 \mathrm{~ms}$. However, the predictive value of the QT interval in predicting the TdP risk is low [21]. Amiodarone is the most effective AAD for rhythm control according to several clinical studies. Although it offers the lowest risk for causing TdP among class III agents, its extracardiac adverse effects limit its long-term use [18]. Several drugs that block the $\mathrm{I}_{\mathrm{Na}}$ current have shown a propensity to induce proarrhythmia $[22,23]$. In general, propafenone use can result in non-TdPmediated ventricular arrhythmias, whereas amiodarone use can cause TdP-associated malignant ventricular arrhythmias [17]. In cases with amiodarone-induced TdP, hypokalemia or hypomagnesemia usually accompanies.

$\mathrm{T}$ waves show ventricular repolarization in ECGs. Given the limited sensitivity of QT dispersion (QTmax - QTmin), the Tp-e interval, Tp-e/QT and Tp-e/QTc ratio have been explored as novel parameters for assessing ventricular repolarization dispersion. Yamaguchi et al [5] demonstrated that the Tp-e interval was a better predictor of TdP than QT dispersion and QTc interval in patients with drug-induced long QT syndrome. Moreover, Liu et al [24] showed that increased QT intervals and Tp-e/QT ratios were associated with drug-induced TdP. In our study, the Tp-e interval, cTp-e interval, Tp-e/QT and Tp-e/QTc ratio parameters were observed to be higher in the amiodarone group than in the propafenone and control groups. These findings may be attributed to the effects of amiodarone on the repolarization phase. No difference was observed in the Tp-e interval, cTp-e interval, Tp-e/QT and Tp-e/QTc ratio parameters between the propafenone and control groups. This indicates that transmural dispersion of the $\mathrm{T}$ wave and variation in the QT interval encompass changes in the repolarization of the action potential. Therefore, these ECG markers may not be adequate to detect all types of drug-induced cardiac 
arrhythmias [8]. The 2016 AF guidelines published by the European Heart Association state that safety should be prioritized in patients requiring prolonged use of AADs [4]. Moreover, some AADs, such as propafenone, which do not prolong the QT interval, can still increase the risk for proarrhythmia [25]. Therefore, new parameters that do not depend solely on the QT interval are needed to help identify the risk for malignant ventricular arrhythmias.

The iCEB is a non-invasive parameter for assessing the risk for drug-induced ventricular proarrhythmia. It accounts for the repolarization and depolarization phases of the action potential and can help identify repolarization dispersion and conduction velocity abnormalities, which are important markers in the pathogenesis of arrhythmia. Several studies have demonstrated the relationship between the cardiac wavelength $\lambda(\lambda=$ effective refractory period (ERP) $\times$ conduction velocity) and malignant ventricular arrhythmias. Aidonidis et al [26] used the cardiac wavelength $\lambda$ as a parameter to study ventricular reentrant tachycardia and $\mathrm{AF}$ in an animal model. Another animal study demonstrated that QT interval was associated with ERP and that changes in QRS duration coincided with changes in conduction velocity [8]. More recently, Robyns et al [9] showed that the ERP measured invasively during electrophysiological study correlated with the QT interval. The study reported that iCEB (QT/QRS) and cardiac wavelength $\lambda(($ ERP $) \times$ conduction velocity $)$ are equal, as each assess the repolarization and depolarization of the action potentials.

Previous studies have suggested that changes in iCEB values may predict an increased susceptibility to malignant ventricular arrhythmias. Lu et al [8] showed that the administration of dofetilide, an $\mathrm{I}_{\mathrm{Kr}}$ blocker, resulted in increased QT, Tp-e intervals and $\mathrm{iCEB}$, causing $\mathrm{TdP}$ in rabbit ventricular wedge samples. In addition, the study showed that encainide, an $\mathrm{I}_{\mathrm{NA}}$ blocker, had no effect on QT and Tp-e intervals, reduced the iCEB values and resulted in non-TdP-like VT. Given these findings, the authors suggested that iCEB may be a better biomarker that $\mathrm{Tp}$-e and $\mathrm{QT}$ intervals for identifying the risk for drug-induced non-TdP, such as ventricular arrhythmia. Robyns et al [9] showed that sotalol administration increases iCEB, whereas flecainide usage decreases iCEB in patients with paroxysmal supraventricular arrhythmias. Additionally, the authors compared iCEB values of 70 genotype positive congenital long QT syndrome (LQTS) patients, 57 genotype positive brugada syndrome $(\mathrm{BrS})$ patients and 65 genotype negative family members in the same study. Their data showed that $\mathrm{iCEB}$ and $\mathrm{iCEBc}$ are significantly increased in LQTS and significantly reduced in $\mathrm{BrS}$ versus genotype negative family members. They attributed the higher iCEB to QT prolongation in LQTS patients. The probable reason for this is that class III agents increase phase 3 of the action potential and ERP by blocking $\mathrm{I}_{\mathrm{Kr}}[18]$. Furthermore, QT interval is prolonged either due to loss of function of the slow $\left(\mathrm{I}_{\mathrm{KS}}\right)$ or the rapid $\left(\mathrm{I}_{\mathrm{Kr}}\right)$ in LQTS [27]. The possible mechanism of reduction of iCEB (QT/QRS) in treatment with class IC drugs is due to the blockage of fast $\mathrm{Na}^{+}$channels. The mechanism of reduction of iCEB in BrS, which causes a loss of function of the cardiac sodium channel, is reduced sodium current. Accordingly, upstroke velocity of phase 0 of the action potential decreases [28]. This is seen as an increase in QRS duration on the surface ECG [9].

In this study, Tp-e and $\mathrm{iCEBc}$ values were highest in the amiodarone group and both $\mathrm{iCEB}$ and $\mathrm{iCEBc}$ values were the lowest in the propafenone group. In addition, the iCEBc value of the patient who presented with TdP was 5.2, while the iCEBc value of the patient who presented with non-TdP mediated VT was 3.58. Further studies are needed to determine whether these electrophysiological changes are associated with ventricular arrhythmias. In addition, iCEB values in patients using amiodarone were similar to those in health controls. Because amiodarone is a multiple ion-channel blocker, the QT/QRS ratio may be unaffected as it prolongs both the QT interval and QRS duration. Given that $\mathrm{iCEB}$ is equal to cardiac wavelength $(\lambda)$, iCEB may be used as an indicator for malign arrhythmias. While AADs that prolong the QT interval cause increased iCEB and iCEBc durations, AADs that prolong the QRS duration result in decreased $\mathrm{iCEB}$ and iCEBc durations. Therefore, iCEBc prolongation and shortening may be an indicator for increased risk for malign arrhythmias.

\section{Study limitations}

This study had several limitations. First, a relatively small sample size was used and only patients receiving amiodarone and propafenone were included. Second, the cross-sectional design precluded our ability to assess causality. Patients were not followed longitudinally to assess the onset clinical events such as malignant ventricular arrhythmia and SCD. Third, ECGs were performed in patients in the cardiology outpatient clinic to assess the potential risk for cardiac arrhythmia; however, $24 \mathrm{~h}$ Holter monitoring may have been more appropriate for drug-related arrhythmia screening. Moreover, comorbidity and heart failure were more common in the amiodarone group. QRS duration, QT and Tp-e intervals may change due to left ventricular remodeling in heart failure as well as comorbidity. These confounding factors limited the evaluation of the pure effect of amiodarone. Finally, the validity of the observed iCEB values was not assessed given the invasive nature of the cardiac wavelength $\lambda$ measurement.

\section{Conclusions}

To the best of our knowledge, this was the first study to investigate $\mathrm{iCEB}$ and $\mathrm{iCEBc}$ parameters in patients with $\mathrm{AF}$. In this study, higher cTp-e intervals, Tp-e/QTc ratios and iCEBc parameters were observed in patients using amiodarone, while $\mathrm{iCEB}$ and $\mathrm{iCEBc}$ values were lowest among patients with $\mathrm{AF}$ using propafenone. Because iCEB and $\mathrm{iCEBc}$ represent the balance between the depolarization and repolarization of the cardiac action potential, these parameters may serve as a noninvasive, simple, and novel biomarker for detecting increased proarrhythmia risk in patients with $\mathrm{AF}$ on antiarrhythmic-drug therapy. Further studies are needed to determine whether these electrophysiological changes are associated with ventricular arrhythmias for patients with $\mathrm{AF}$ on antiarrhythmic-drug therapy. 


\section{Acknowledgments}

None to declare.

\section{Financial Disclosure}

This study received no grant funding from any agency in the public, commercial or not-for-profit sectors.

\section{Conflict of Interest}

The authors declare that they have no conflict of interest.

\section{Informed Consent}

A signed informed written consent form was obtained from all participants.

\section{Author Contributions}

AA, RA and AS performed the research and analysed data. MK, RA, AA, EA and AS provided clinical data. AA, RA and MK wrote the paper and all authors critically reviewed and edited the paper. All authors read and approved the manuscript.

\section{Data Availability}

The data used to support the findings of this study are available from the corresponding author upon request.

\section{References}

1. Stewart S, Hart CL, Hole DJ, McMurray JJ. A population-based study of the long-term risks associated with atrial fibrillation: 20-year follow-up of the Renfrew/Paisley study. Am J Med. 2002;113(5):359-364.

2. Andrade J, Khairy P, Dobrev D, Nattel S. The clinical profile and pathophysiology of atrial fibrillation: relationships among clinical features, epidemiology, and mechanisms. Circ Res. 2014;114(9):1453-1468.

3. Lafuente-Lafuente C, Valembois L, Bergmann JF, Belmin J. Antiarrhythmics for maintaining sinus rhythm after cardioversion of atrial fibrillation. Cochrane Database Syst Rev. 2015;3:CD005049.

4. Kirchhof P, Benussi S, Kotecha D, Ahlsson A, Atar D, Casadei B, Castella M, et al. 2016 ESC Guidelines for the management of atrial fibrillation developed in collaboration with EACTS. Eur J Cardiothorac Surg. 2016;50(5):e1-e88.

5. Yamaguchi M, Shimizu M, Ino H, Terai H, Uchiyama K, Oe K, Mabuchi T, et al. T wave peak-to-end interval and
QT dispersion in acquired long QT syndrome: a new index for arrhythmogenicity. Clin Sci (Lond). 2003;105(6):671676.

6. Lubinski A, Lewicka-Nowak E, Kempa M, Baczynska AM, Romanowska I, Swiatecka G. New insight into repolarization abnormalities in patients with congenital long QT syndrome: the increased transmural dispersion of repolarization. Pacing Clin Electrophysiol. 1998;21(1 Pt 2):172-175.

7. Castro-Torres Y, Carmona-Puerta R, Katholi RE. Ventricular repolarization markers for predicting malignant arrhythmias in clinical practice. World J Clin Cases. 2015;3(8):705-720.

8. Lu HR, Yan GX, Gallacher DJ. A new biomarker-index of cardiac electrophysiological balance (iCEB) — plays an important role in drug-induced cardiac arrhythmias: beyond QT-prolongation and Torsades de Pointes (TdPs). J Pharmacol Toxicol Methods. 2013;68(2):250-259.

9. Robyns T, Lu HR, Gallacher DJ, Garweg C, Ector J, Willems R, Janssens S, et al. Evaluation of Index of CardioElectrophysiological Balance (iCEB) as a New Biomarker for the Identification of Patients at Increased Arrhythmic Risk. Ann Noninvasive Electrocardiol. 2016;21(3):294304.

10. January CT, Wann LS, Alpert JS, Calkins H, Cigarroa JE, Cleveland JC, Conti JB, et al. 2014 AHA/ACC/HRS guideline for the management of patients with atrial fibrillation: executive summary: a report of the American College of Cardiology/American Heart Association Task Force on practice guidelines and the Heart Rhythm Society. Journal of the American College of Cardiology. 2014;64(21):2246-2280.

11. Lip GY, Nieuwlaat R, Pisters R, Lane DA, Crijns HJ. Refining clinical risk stratification for predicting stroke and thromboembolism in atrial fibrillation using a novel risk factor-based approach: the euro heart survey on atrial fibrillation. Chest. 2010;137(2):263-272.

12. Lang RM, Badano LP, Mor-Avi V, Afilalo J, Armstrong A, Ernande L, Flachskampf FA, et al. Recommendations for cardiac chamber quantification by echocardiography in adults: an update from the American Society of Echocardiography and the European Association of Cardiovascular Imaging. Eur Heart J Cardiovasc Imaging. 2015;16(3):233-270.

13. Van Gelder IC, Hagens VE, Bosker HA, Kingma JH, Kamp O, Kingma T, Said SA, et al. A comparison of rate control and rhythm control in patients with recurrent persistent atrial fibrillation. N Engl J Med. 2002;347(23):18341840.

14. Wyse DG, Waldo AL, DiMarco JP, Domanski MJ, Rosenberg Y, Schron EB, Kellen JC, et al. A comparison of rate control and rhythm control in patients with atrial fibrillation. N Engl J Med. 2002;347(23):1825-1833.

15. Ogawa S, Yamashita T, Yamazaki T, Aizawa Y, Atarashi $\mathrm{H}$, Inoue $\mathrm{H}$, Ohe $\mathrm{T}$, et al. Optimal treatment strategy for patients with paroxysmal atrial fibrillation: J-RHYTHM Study. Circ J. 2009;73(2):242-248.

16. Dagres N, Lewalter T, Lip GY, Pison L, Proclemer A, Blomstrom-Lundqvist C, Scientific Initiatives Com- 
mittee EHRA. Current practice of antiarrhythmic drug therapy for prevention of atrial fibrillation in Europe: The European Heart Rhythm Association survey. Europace. 2013;15(4):478-481.

17. Nattel S, Gersh BJ, Opie LH. Antiarrhythmic drugs and strategies. In: Drugs for the heart. Saunders Elsevier Inc., Philadelphia; 2013. p. 272-331.

18. Dan GA, Martinez-Rubio A, Agewall S, Boriani G, Borggrefe M, Gaita F, van Gelder I, et al. Antiarrhythmic drugs-clinical use and clinical decision making: a consensus document from the European Heart Rhythm Association (EHRA) and European Society of Cardiology (ESC) Working Group on Cardiovascular Pharmacology, endorsed by the Heart Rhythm Society (HRS), Asia-Pacific Heart Rhythm Society (APHRS) and International Society of Cardiovascular Pharmacotherapy (ISCP). Europace. 2018;20(5):731-732an.

19. Zimetbaum P. Antiarrhythmic drug therapy for atrial fibrillation. Circulation. 2012;125(2):381-389.

20. Drew BJ, Ackerman MJ, Funk M, Gibler WB, Kligfield P, Menon V, Philippides GJ, et al. Prevention of torsade de pointes in hospital settings: a scientific statement from the American Heart Association and the American College of Cardiology Foundation. J Am Coll Cardiol. 2010;55(9):934-947.

21. Ramalho D, Freitas J. Drug-induced life-threatening arrhythmias and sudden cardiac death: A clinical perspective of long QT, short QT and Brugada syndromes. Rev Port Cardiol. 2018;37(5):435-446.
22. Gintant GA, Gallacher DJ, Pugsley MK. The 'overlysensitive' heart: sodium channel block and QRS interval prolongation. Br J Pharmacol. 2011;164(2):254-259.

23. Harmer AR, Valentin JP, Pollard CE. On the relationship between block of the cardiac $\mathrm{Na}(+)$ channel and drug-induced prolongation of the QRS complex. Br J Pharmacol. 2011;164(2):260-273.

24. Liu T, Brown BS, Wu Y, Antzelevitch C, Kowey PR, Yan GX. Blinded validation of the isolated arterially perfused rabbit ventricular wedge in preclinical assessment of drug-induced proarrhythmias. Heart Rhythm. 2006;3(8):948-956.

25. Laverty H, Benson C, Cartwright E, Cross M, Garland C, Hammond T, Holloway C, et al. How can we improve our understanding of cardiovascular safety liabilities to develop safer medicines? Br J Pharmacol. 2011;163(4):675693.

26. Aidonidis I, Poyatzi A, Stamatiou G, Lymberi M, Stamatoyannis N, Molyvdas PA. Dose-related shortening of ventricular tachycardia cycle length after administration of the KATP channel opener bimakalim in a 4-day-old chronic infarct anesthetized pig model. J Cardiovasc Pharmacol Ther. 2009;14(3):222-230.

27. Schwartz PJ, Crotti L, Insolia R. Long-QT syndrome: from genetics to management. Circ Arrhythm Electrophysiol. 2012;5(4):868-877.

28. King JH, Huang CL, Fraser JA. Determinants of myocardial conduction velocity: implications for arrhythmogenesis. Front Physiol. 2013;4:154. 